\title{
Aplicação preliminar do método QRAM para avaliação de riscos para segurança ocupacional na construção civil
}

\author{
Abel Pinto ${ }^{\text {**}}$, lsabel Nunes ${ }^{\mathrm{b}}$, Rita Ribeiroc ${ }^{\mathrm{c}}$, Luis Carlos Paschoarelli ${ }^{\mathrm{d}}$ \\ a*abel.fnpinto@gmail.com, UNL, Portugal \\ bimn@fct.unl.pt, UNL, Portugal \\ crar@uninova.pt, UNINOVA, Portugal \\ dpaschoarelli@faac.unesp.br, UNESP, Brasil
}

\begin{abstract}
Resumo
A segurança ocupacional é imprescindível na indústria da construção civil e a análise e avaliação de riscos para a segurança ocupacional (AARSO) é o primeiro e fundamental passo para alcançá-la, baseado na definição e implementação de programas de prevenção. A AARSO é um processo complexo, que implica a consideração e análise de muitos parâmetros quantitativos e/ou qualitativos que são difíceis de quantificar. As metodologias AARSO utilizadas na indústria da construção civil são baseadas em informação sujeita a incerteza (sendo tratada por técnicas probabilísticas e/ou estatísticas), difusa, imprecisa e/ou incompleta. lsso implica algumas limitações, como, por exemplo, obrigar os analistas a estimar parâmetros ou efetuar comparações com outros canteiros de obras (o que afasta do sistema real em estudo). 0 objetivo inicial deste estudo foi efetuar a pré-validação de um método AARSO, o QRAM, em duas cidades brasileiras, de médio e grande porte.
\end{abstract}

Palavras-chave

Avaliação de risco. Segurança ocupacional. Conjuntos difusos. Indústria da construção. Validação.

\section{Introdução}

Devido às suas características e problemas específicos (DUARTE; CORDEIRO, 1999; FREJ; ALENCAR, 2010; GUERRINI; VERGNA, 2011), a indústria da construção civil registra elevados índices de sinistros ocupacional (TAM; ZENG; DENG, 2004; MELIÁ et al., 2008; INSTITUTO..., 2008; HYOUNG et al. 2009), com custos econômicos elevados e que afetam a produtividade do setor (SILVERSTEIN et al., 1998; DONG et al., 2007; CAMBRAIS; SAURIN; FORMOSO 2008).

A integração dos requisitos de segurança na etapa de desenvolvimento de produto (projeto) parece ser uma das alternativas com maior potencial de criar benefícios, uma vez que pode eliminar ou reduzir os perigos nas suas origens, dado que as falhas de projeto são uma das mais importantes categorias de causa-raiz dos acidentes nessa indústria (SAURIN, 2005).
A análise e avaliação de riscos para a segurança ocupacional (AARSO) é o primeiro e fundamental passo para alcançar essa integração, permitindo a definição e implementação de programas de prevenção logo no início da construção.

Diversos estudos (DEDOBBELEER; BELAND, 1991; RINGEN et al., 1995; GILLEN et al., 1997; LAITINEN; MARJAMAKI; PAIVARINTA, 1999; LOOSEMORE; LEE, 2001; TAM; ZENG; DENG, 2004) identificaram algumas das causas que afetam o nível de segurança em canteiros da construção civil, das quais se destacam:

- Inadequada organização do trabalho;

- Inadequada gestão da segurança;

- Falta de formação, informação e sensibilização;

- Fraca cultura de segurança;

- Falta de coordenação e de supervisão; 
- Pressões econômicas e cronológicas;

- Comunicação, interna e externa, ineficaz;

- Fraco envolvimento dos trabalhadores e outras partes interessadas nas questões de segurança;

- Dinâmica do ato de construir com constantes alterações e mudanças;

- Diversidade de empregadores e profissionais a trabalhar simultaneamente no canteiro de obra;

- Rotatividade ocupacional;

- Operação de equipamento pesado e trabalho em elevadas alturas;

- Jornadas de trabalho longas e hábitos de vida pouco saudáveis;

- Cadeias de subcontratação alongadas;

- Comparativamente com outras indústrias, os trabalhadores são mais responsáveis pela organização do seu posto de trabalho e pela sua segurança;

- Fraco envolvimento da direção em questões de segurança e dificuldade em atribuir recursos à segurança;

- Trabalho duro e, socialmente, pouco digno;

- Locais de trabalho afastados dos locais de residência, com a consequente deslocação dos trabalhadores.

0 processo de AARSO envolve essencialmente a identificação dos perigos, análise, caracterização, avaliação e hierarquização dos riscos e a definição de medidas de prevenção e/ou proteção. É um processo complexo, que implica no estudo e estimativa da magnitude de um vasto conjunto de fatores que são, por vezes, difíceis de quantificar/qualificar, com o objetivo de conhecer as vulnerabilidades do sistema em análise. A informação a recolher e a analisar é respeitante a:

- Processos construtivos - como se faz, com que meios humanos e materiais;

- Estrutura hierárquica utilizada no processo - cadeia de comando;

- Dados históricos de ocorrências relacionadas com a segurança ocupacional - acidentes e incidentes já ocorridos no canteiro de obras ou em canteiros similares;

- Objetivos a atingir - aceitação do nível de risco;

- Limites financeiros para o investimento a efetuar-em função dos objetivos propostos e da viabilidade técnico-econômica das soluções propostas que visam atingir os objetivos estabelecidos.

Algumas especificidades e crenças inerentes à cultura própria do setor da construção civil levam a que as metodologias AARSO sejam pouco usadas. Akintoye e Macleod (1997) apontam algumas das principais causas:
- A reduzida familiaridade com as metodologias AARSO disponíveis;

- 0 grau de sofisticação da maioria das metodologias torna difícil a sua aplicação ao setor da construção civil;

- A falta de tempo, de conhecimentos e de informação confiável para aplicar eficazmente as metodologias;

- As dúvidas acerca da aplicabilidade das metodologias ao setor da construção civil;

- A pequena dimensão da maioria das obras não permite alocar os recursos necessários à AARSO (inexistência de recursos humanos e escassez de recursos financeiros);

- Os riscos são considerados subjetivos e são avaliados com base na experiência passada (percepção);

- Os benefícios resultantes da análise de riscos não são facilmente perceptíveis;

- A AARSO não constitui, habitualmente, obrigação contratual.

Existem diversas metodologias AARSO descritas na literatura (AVEN, 2003; COOPER et al., 2006; HAMMER, 2001; HOLLNAGEL, 2008; LOOSEMORE et al., 2006; RINGDAHL, 2001). Essas metodologias são baseadas em informações sujeitas a incerteza, imprecisão, ambiguidade e, normalmente, incompleta. Destes fatos resultam limitações (KARWOWSKI; MITAL, 1986; CORNELL, 1996; WANG; MCCAULEY-BELL, 1997; PENDER, 2001; SII; WANG; RUXTON, 2001; TIXIER et al., 2002; FABER; STEWART, 2003; NILSEN; AVEN, 2003; KENTEL; ARAL, 2004), das quais destacam-se como mais relevantes:

- Os sistemas humanos são inerentemente imprecisos;

- A dificuldade de quantificar as consequências dos perigos devido aos elevados níveis de incerteza envolvidos;

- A necessidade de efetuar estimativas, julgamentos e levantamentos torna o processo muito dependente da capacidade do(s) analista(s), até para interpretar os resultados;

- Os empreendimentos de construção civil são únicos, o que reduz a relevância e a confiabilidade da utilização de técnicas estatísticas.

Diante do exposto, o objetivo principal deste estudo foi pré-validar um método qualitativo para AARSO, doravante denominado QRAM (Qualitative Risk Assessment Method), adequado à indústria da construção civil. Trata-se de uma pré-validação, uma vez que pretende-se validar exclusivamente os fatores que influenciam quatro formas de acidentes específicos, a saber: queda (em altura e ao mesmo nível), soterramento, contato eléctrico e afogamento.

Para tratar da incerteza dos fatores considerados no QRAM, aplica-se a teoria dos conjuntos difusos (ZADEH, 
1965), uma vez que possibilita a representação, modelação e tratamento da incerteza e imprecisão intrínsecas à modelação dos riscos para a segurança ocupacional.

0 objetivo deste trabalho é apresentar as principais características de um novo método AARSO, o QRAM, que está em desenvolvimento, recolher e analisar os dados coletados no teste de aplicação efetuado em canteiros de construção localizados em duas cidades brasileiras, de médio e grande porte, discutir os resultados obtidos quanto às dificuldades e limitações do processo AARSO na indústria da construção e concluir acerca das vantagens do QRAM para ultrapassar essas dificuldades.

\section{Enquadramento teórico}

Os acidentes de trabalho na indústria da construção ocorrem principalmente devido a: 1) falta de sensibilização ou de formação; 2) falta de supervisão; 3) falta de meios para realizar a tarefa com segurança; 4) erro de julgamento; 5) descuido; 6) apatia; 7) imprudência; ou 8) condição insegura (SAWACHA; NAOUM; FONG, 1999; ABDELHAMID, 2000).

Modelar os fatores de risco na segurança ocupacional é um fenômeno complexo (CHOUDHRY; FANG; MOHAMED, 2007). As abordagens AARSO tradicionais são baseadas no uso de estatística e probabilidades para o tratamento dos dados. Como a informação disponível no setor da construção civil é imprecisa, difusa e incompleta, particularmente na fase de projeto, tal abordagem não produz resultados adequados (APELAND; AVEN; NILSEN, 2002). 0 uso de técnicas probabilísticas e estatísticas pode mascarar aspectos do conhecimento incompleto e impreciso, produzindo uma falsa sensação de exatidão e precisão e conduzindo a decisões enviesadas e incorretas (ANDERSSON, 1986; FABER; STEWART, 2003; NILSEN; AVEN, 2003).

A segurança depende de fatores técnicos, humanos e organizacionais. Algumas explicações apresentadas para os altos índices de acidentes na construção civil têm incluído fatores organizacionais como o estilo de gestão e a política de segurança da empresa. Incluem-se neste caso as características pessoais como a idade, a experiência, o conhecimento, a percepção do risco, a confiança interpares e a motivação (LANDEWEERD et al., 1990, SAURIN; RIBEIRO, 2000). As falhas humanas estão na gênese de cerca de 95\% dos acidentes industriais (CORREA; CARDOSO JUNIOR, 2007). 0 Health and Safety Executive (HSE, 2002) concluiu que o comportamento humano é o fator que contribui para cerca de $80 \%$ dos acidentes de trabalho no setor da construção civil. A erosão e degradação dos sistemas de gestão da segurança são, nesse setor, muito provavelmente devido à existência de procedimentos impraticáveis, à insuficiente manutenção de máquinas, equipamentos e barreiras de segurança, à coexistência de objetivos conflituosos, a falhas nos procedimentos de comunicação e à inadequada ou insuficiente formação/sensibilização (TRBOJEVIC, 2008).

No entanto, esse conjunto de fatores não se reflete nos resultados das avaliações de risco. Assim, os resultados das AARSO são parciais, porque só revelam os riscos derivados de fatores técnicos e podem ser enviesados, devido à má "qualidade" da informação disponível. A grande limitação para a inclusão de fatores organizacionais e humanos é a ausência de consenso sobre o conjunto de parâmetros relacionados com o comportamento para a segurança, a organização de segurança, a organização do trabalho, a supervisão, a liderança, os fatores pessoais, a comunicação e consulta e a forma como estes afetam o desempenho da segurança, especialmente em ambientes "turbulentos”, como é o caso da construção civil. Além disso, a forma e os critérios de avaliação que se encontram descritos na literatura (GULDENMUND, 2000; MEARNS; FLIN, 1999) não são facilmente praticáveis.

A proposta do QRAM é compreender os riscos existentes, nas suas várias vertentes, e hierarquizá-los, discriminando o contributo dos vários fatores de risco, a fim de melhor planejar e desenvolver medidas para os minimizar e/ou controlar.

\section{0 modelo QRAM}

Face ao exposto, torna-se evidente que a AARSO, no setor da construção civil, tem de ultrapassar a insuficiência, a ambiguidade e a imprecisão dos dados disponíveis que resultam, quer de informação mal definida, incompleta e, por vezes, conflituosa (especialmente na fase de concepção), quer por falta de entendimento dos analistas na interpretação das informações disponíveis. Além disso, o uso de técnicas probabilísticas e estatísticas pode mascarar outros aspectos do conhecimento incompleto e impreciso, levando a uma falsa sensação de exatidão e precisão e conduzindo a decisões incorretas.

0 uso da teoria dos conjuntos difusos (fuzzy set theory) (ZADEH, 1965) parece ser uma abordagem mais adequada para representar, de forma mais realista, os riscos ocupacionais. Apresenta maneira natural de modelar a incerteza e imprecisão intrínsecas aos sistemas e permite a inclusão da criatividade humana e da intuição, que é um ingrediente essencial para 
a fase da análise e avaliação dos riscos (RU; ELOFF, 1996), ou seja, determinar quais os fatores que contribuem e com qual magnitude do risco. Uma abordagem que a lógica difusa possibilita para lidar com imprecisão linguística é a utilização de variáveis linguísticas (VL), em vez de valores numéricos.

0 apreço pela lógica difusa é partilhado por diversos autores: Andersson (1986), Liu et al. (2004), Nunes (2005) e Mure, Demichela e Piccinini, (2006). Existem alguns trabalhos que aplicam a teoria dos conjuntos difusos às áreas dos fatores humanos, ergonomia e avaliação de riscos, com destaque para aqueles descritos por Karwowski e Mital (1986), Kangari e Riggs (1989), McCauley-Bell e Badiru (1996), Carr e Tah (2001), Tam, Zeng e Deng (2002), Hanson, Redfern e Mazumdar, (2003), Lee e Halpin (2003), Nunes (2003), Nunes (2005), Nunes (2005), Karwowski e Mital (1986), Oke et al. (2006), Dagdeviren e Ihsan (2008), Yang, Bonsall e Wang (2008), Azadeh et al. (2008) e Gurcanli e Mungen (2009).

0 modelo QRAM baseia-se em dados reais e não em estimativas. Os dados são obtidos através da observação direta da realidade existente nos locais de trabalho, entrevistas a trabalhadores, encarregados e engenheiros responsáveis e consulta da documentação do canteiro de obras (procedimentos de trabalho, relatórios de investigação de incidentes/acidentes, atas de reunião de obra....). Os dados devem ser transformados e normalizados utilizando lógica difusa e serão agregados com recurso a operadores de média, com o objetivo de avaliar e hierarquizar os riscos existentes na obra.

\subsection{Modelo conceptual do QRAM}

Assim, o risco é definido em função de quatro dimensões (ver expressão 1): fatores principais $\left(F_{p}\right)$, fatores adicionais $\left(F_{A}\right)$,cumprimento das prescrições de segurança $\left(C_{S}\right)$ e barreiras de segurança $\left(B_{S}\right)$. 0 nível de risco é função dessas quatro dimensões. Os $\mathrm{F}_{\mathrm{p}}$ modelam a gravidade dos danos no estado de saúde do(s) acidentado(s), os $F_{A}$ modelam a possibilidade de ocorrência dos acidentes de trabalho e as $B_{S}$ modelam a eficácia das medidas de segurança, implementadas para reduzir as consequências e/ou a possibilidade de ocorrência dos acidentes de trabalho. 0 cumprimento das prescrições de segurança, $C_{S}$, não se refere a uma forma de contato em particular, sendo comum a todas as formas de contato. É transversal a todos os perigos identificados no canteiro de obra, ou seja, modela o empenho de toda a cadeia hierárquica na gestão da segurança, e o respeito por suas prescrições.

$$
R=f\left(F_{P}, F_{A}, C_{S}, B_{S}\right)
$$

\subsection{Tratamento da incerteza no QRAM}

No QRAM, a AARSO é baseada em dados reais. Nesta matéria (como na maioria dos problemas reais), os dados (variáveis de entrada) não são dicotômicos nem determinísticos, pelo que não podem ser tratados de forma precisa, e a descrição detalhada de um sistema real requer muito mais dados do que a capacidade de um ser humano consegue, em simultâneo, reconhecer, processar e compreender (ZIMMERMAN, 1993). Portanto, a aplicação da teoria dos conjuntos difusos pode trazer as seguintes vantagens, relativamente às formas de modelação tradicionais:

- Requer menos regras;

- Mais variáveis observáveis podem ser valoradas;

- 0 uso de variáveis linguísticas nos deixa mais perto do pensamento humano;

- Proporciona um rápido protótipo dos sistemas;

- Simplifica a aquisição do conhecimento.

Um modelo de análise e avaliação difuso deve incluir os processos de identificação, quantificação e combinação de variáveis para criar um modelo conceitual de decisão em ambiente difuso. 0 uso de conjuntos difusos num modelo implica (MURE et al., 2006):

- Escolha das variáveis de input e sua representação como variáveis difusas de entrada (fuzificação);

- Definição das relações entre as variáveis de entrada para obter as variáveis de saída (resultados/modelo conceitual);

Normalização e agregação dos fatores com operadores especializados.

\section{Teoria dos conjuntos difusos}

A teoria dos conjuntos difusos (TCD) é uma teoria matemática (precisa) para lidar com a imprecisão e o raciocínio aproximado (ZADEH, 1965, 1978, 1983, 1986). Mais especificamente, a lógica difusa pode ser encarada como uma tentativa de formalização de duas notáveis capacidades humanas: 1) a capacidade de conversar, raciocinar e tomar decisões racionais em ambiente de imprecisão, incerteza, informações incompletas, informações contraditórias e parcialidade da verdade, e 2) a capacidade de tratar uma grande variedade de dadossem efetuar medições quantitativas.

0 principal conceito da TCD é o conjunto difuso. Um conjunto difuso (ZIMMERMAN, 1993) apresenta uma fronteira com um contorno gradual, difuso, em contraste com os conjuntos clássicos, que têm fronteiras rígidas. Formalmente, seja $U$ um universo de discurso (domínio) e $u$ um elemento genérico de $U$. Um subconjunto fuzzy $A$, definido em $U$, é um conjunto de pares ordenados: 


$$
A=\{(u, \mu A(u)) \mid u \in U\}
$$

onde $\mu A(u)$ é designado como o grau de pertença de $u$ em $A$. A função de pertença associa a cada elemento $u$ de $U$, um número real $\mu A(u)$, no intervalo $[0,1]$, que representa a transição gradual de membro para não membro desse conjunto difuso.

Os conjuntos difusos podem ser contínuos ou discretos (ZIMMERMAN, 1993), sendo que a forma da função de pertença contém informação acerca do fenômeno em estudo, pelo que a sua escolha terá de ser adequada (descrevendo a evolução) da variável em estudo.

Por exemplo, no QRAM o conjunto difuso que modela o fator principal (gravidade dos danos no estado de saúde do acidentado) para o movimento vertical, esmagamento sobre ou contra (resultado de queda), em função da altura, será definido pelo conjunto difuso contínuo apresentado na Figura 1, o qual foi desenvolvido com base em conhecimento empírico de seis especialistas em segurança ocupacional na indústria da construção civil.

Como se pode observar na Figura 1, o conjunto difuso é definido por funções lineares por troços, onde o domínio da variável gravidade da queda é: [0-220] (cm) com o correspondente valor de pertença no eixo dos Y. Por exemplo, o par ordenado (100, $0.70)$ refere que para uma altura de $100 \mathrm{~cm}$, sendo a zona de impacto (onde o corpo acidentado irá embater) lisa, o valor de pertença ao conjunto difuso "movimento vertical, esmagamento sobre ou contra (resultado de queda)" é 0.7. No caso de na zona de embate existirem objetos com arestas ou ferros em espera desprotegidos, os valores de pertença serão

Movimento vertical, esmagamento sobre ou contra (resultado de queda)

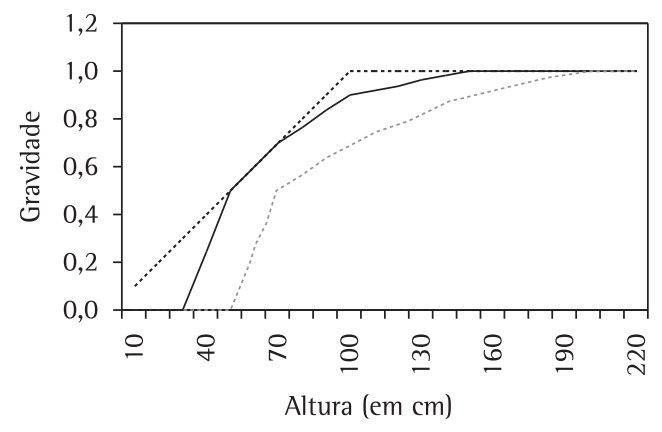

Zona de impacto lisa

Zona de impacto com arestas

....... Zona de impacto com ferros em espera

Figura 1. Gravidade da forma de acidente "movimento vertical, esmagamento sobre ou contra (resultado de queda)" de 0.9 e 1 , respectivamente, valores que representam consequências (gravidade) mais graves.

Outro aspecto interessante da TCD é a definição de operadores lógicos e aritméticos para proceder a operações com os conjuntos difusos. Basicamente existem quatro classes de operadores para agregar variáveis e chegar a conclusões: a) as intersecções; b) as uniões; c) a negação; e d) operadores de agregação (ZIMMERMAN, 1993). Por exemplo, se quisermos exprimir quantos homens altos "e" obesos existem em determinado conjunto, utilizamos a intersecção. Se quisermos exprimir qual o número nesse conjunto de pessoas magras, utilizamos a negação dos obesos, entre outros. No presente estudo, propôs-se utilizar essencialmente os últimos, operadores de agregação, pois o objetivo é agregar as avaliações de cada um dos fatores para determinar o risco global ou parcial. Assim, a agregação entre os diversos parâmetros de risco será efetuada com recurso de média como, por exemplo, a média harmônica.

Segundo Bellman e Zadeh (1970), “a tomada de decisões em contexto real ocorre em ambiente no qual as metas, os constrangimentos e as consequências de eventuais ações não são conhecidas com precisão". Este é o caso do risco no setor da construção civil, pois é necessário agregar diversos fatores imprecisos como, por exemplo, o fator gravidade das consequências (ver Figura 1), para determinar o risco de acidente.

\section{Teste do modelo}

De acordo com Kohn (1995), a validação de um modelo tem por objetivo demonstrar que está isento de erros matemáticos (consistência interna), que não é sensivel aos pequenos erros (mas é sensível aos grandes) ou a incertezas nos dados de entrada (confiabilidade e robustez) e que reproduz o comportamento prático do sistema em estudo (consistência externa). Para ser válido o modelo tem de representar de forma realista o sistema em estudo. Só com o modelo devidamente validado, em variadas e diferentes situações que possam ocorrer no sistema real, os seus resultados são aceites pela comunidade de utilizadores.

Para realizar a pré-validação do QRAM, por ser uma primeira validação e porque a aplicação integral e completa do método num canteiro de construção poderia tomar um tempo além do necessário, optou-se por analisar e avaliar apenas quatro formas de acidente: queda (em altura e ao mesmo nível), soterramento, choque elétrico e afogamento. 0 método foi aplicado em 9 canteiros de construção civil de 5 empresas e localizados em Bauru, cidade de médio porte no interior do estado de São Paulo; e em 4 canteiros de 2 empresas localizadas em Curitiba, capital e cidade 
de grande porte do estado do Paraná. Em nenhum dos canteiros foi possível validar a forma de acidente afogamento por não existir o perigo relacionado.

0 método foi explicado aos responsáveis de segurança dos canteiros que acompanharam a aplicação do método.

\subsection{Questionário}

Para registrar e sistematizar os dados recolhidos, foi elaborado um questionário (ver Anexo 1) dividido em página de rosto e mais seis partes, nomeadamente: 1) página de rosto -apresentação sumária do QRAM e objetivos do questionário; 2) caracterização do especialista em SST; 3) caracterização da empresa (dimensão e tipo de obras que constrói); 4) caracterização do processo de AARSO (quem faz, que metodologias são utilizadas e conhecimento das metodologias, informações utilizadas, fontes de informação, fase(s) da obra e periodicidade com que é realizada a AARSO e importância atribuída pelas hierarquias e trabalhadores aos resultados da AARSO); 5) caracterização da eficácia do processo de AARSO usado na empresa (usabilidade, eficácia, tempo ido e dificuldades percebidas no processo de AARSO); 6) caracterização da eficácia do novo método de AARSO, o QRAM (os mesmos parâmetros); e 7) análise comparativa do novo método, o QRAM (procura recolher a opinião dos especialistas acerca da facilidade de aplicação, rapidez de aplicação e confiabilidade dos resultados obtidos com o QRAM, comparativamente aos métodos usualmente utilizados pelos especialistas).

\subsection{Análise dos resultados obtidos com o questionário}

Os 13 questionários analisados permitem concluir que das 5 empresas contatadas em Bauru (SP), apenas 2 tinham serviços internos de saúde e segurança no trabalho, nas 3 restantes, 1 tem um técnico de SST que acumula essa função com outras funções administrativas, as 2 restantes quando têm qualquer problema na área da SST contratam um engenheiro de segurança para avaliação $a d-h o c ;$ as 2 empresas contatadas em Curitiba (PR) tinham serviços internos de saúde e segurança no trabalho. Seis das empresas constroem edificações (habitacionais, comerciais e escritórios) e uma dedica-se à construção de infraestruturas de telecomunicações.

Todos os 4 canteiros analisados em Curitiba tinham PCMAT (Programa de Condições e Meio Ambiente de Trabalho na Indústria da Construção). Dos 9 canteiros analisados em Bauru, apenas 2 tinham PCMAT.
Nos restantes 7, os riscos iam sendo analisados e controlados de forma ad-hoc, ou seja, dependendo da experiência e percepção dos riscos dos responsáveis da segurança, engenheiros chefes (em 4 dos 7 canteiros) e mestres de obra (em 3 dos 7 canteiros). Nesses 7 canteiros, foi respondido que nem a direção das empresas nem os trabalhadores consideram importante a análise de riscos laborais.

Nos 6 canteiros com PCMAT, 5 usaram o método APP (análise preliminar de perigos) e uma auditoria com check-list. As AARSO são efetuadas no início das obras, não havendo uma periodicidade definida para a reavaliação dos riscos. Os técnicos entrevistados tomaram conhecimento das metodologias AARSO em módulos de cursos de SST (saúde e segurança no trabalho). Nenhum dos entrevistados teve formação específica em metodologias AARSO.

Nas empresas com serviços internos de SST, quem realiza a AARSO são os técnicos de SST; nas restantes é, normalmente, o engenheiro chefe e, sendo um processo pouco sistemático e $a d-h o c$, não há uma fase da obra nem uma periodicidade definidas para a sua realização.

Todos os 13 inquiridos responderam que, no processo de AARSO, a informação utilizada é referente aos equipamentos e materiais. Em 7 dos canteiros foi igualmente relatado que utilizam informação do projeto de construção. Todos referiram que usam a experiência de obras anteriores para realizar a AARSO.

Só em 3 dos canteiros os trabalhadores são consultados no processo de AARSO. Em 9 só o mestre de obras é consultado, e num dos canteiros o engenheiro chefe realiza a AARSO sem consultar ninguém.

Quanto às dificuldades sentidas na realização do processo de AARSO, 3 dos entrevistados responderam que a maior dificuldade é a falta de colaboração das chefias (incluindo a direção das empresas), 4 referiram a falta de dados/informação e os restantes 6 não relataram qualquer dificuldade.

Nas entrevistas efetuadas, 10 dos entrevistados consideraram os "métodos" utilizados pouco eficazes e 3 acharam os métodos utilizados, APP (2) e auditoria com check-list (1), como eficazes.

Só em 1 dos canteiros, os fatores ergonômicos e psicossociais são considerados na AR, embora de forma pouco consistente e pouco sistemática.

A falta de avaliações e análises de riscos na maior parte dos canteiros de construção (7 em 13) e a utilização de metodologias AARSO pouco adequadas nos restantes foram as maiores dificuldades sentidas neste estudo porque não foi possível comparar os resultados obtidos através do QRAM com os resultados obtidos com outros métodos, o que inviabilizou o seu objetivo inicial. 
Em todos os canteiros contatados, a forma de comunicar os riscos aos trabalhadores é através de palestras.

A falta de AARSO nos canteiros de construção civil torna a minimização e controle dos riscos muito pouco eficiente e eficaz. A falta de análise e avaliação dos riscos não permite o correto conhecimento dos riscos e respectivas características, pelo que as medidas de prevenção/proteção observadas nos 7 canteiros sem PCMAT são, de modo geral, inadequadas e desajustadas da realidade. A falta de acompanhamento das obras por técnicos de SST tem, por consequência, a falta de cultura de segurança e da percepção do risco, porque os trabalhadores e hierarquias não têm formação adequada nos domínios da SST e não estão sensibilizados para a importância de trabalhar com segurança, o que pode explicar a pouca importância que é dada aos resultados da AARSO.

\section{Considerações finais}

0 estudo realizado, devido às contingências relatadas (alta de AR nos canteiros de obras, efetuada com recurso a metodologias AR, comprovadas e descritas na literatura), não permitiu alcançar os objetivos iniciais, a pré-validação do QRAM, no entanto os testes efetuados foram importantes para perceber a aceitação do método e testar a sua aplicabilidade.

Pode-se concluir que o QRAM foi considerado muito interessante pelos técnicos entrevistados, quer pela consistência e abrangência dos resultados obtidos, quer pela facilidade da sua aplicação.

Nas três formas de acidente analisadas, a gravidade foi estimada com maior consistência do que é realizado habitualmente, e os fatores que podem conduzir ao acidente foram identificados e caracterizados de forma mais completa do que habitualmente.

No futuro, o QRAM tem de ser validado para todas as formas de acidente e em vários canteiros, com obras de dimensão e tipologias variadas.

\section{Referências}

ABDELHAMID, J. G. Identifying root cause of construction accidents. Journal of Construction Engineering and Management, v. 1, n. 126, p. 52-60, 2000. http://dx.doi. org/10.1061/(ASCE)0733-9364(2000)126:1(52)

AKINTOYE, A. S.; MacLEOD, M. J. Risk analysis and management in construction. International Journal of Management, v. 1, n. 15, p. 31-38, 1997.

ANDERSSON, L. A new method based on the theory of fuzzy sets to obtaining an indication of risk. Civil Engineering and Environmental Systems, v. 3, n. 3, p. 164-174, 1986. http://dx.doi.org/10.1080/02630258608970440

APELAND, S.; AVEN, T.; NILSEN, T. Quantifying uncertainty under a predictive, epistemic approach to risk analysis. Reliability Engineering and System Safety, v. 75, p. 93-102, 2002. http://dx.doi.org/10.1016/ S0951-8320(01)00122-3

AVEN, T. Foundations of Risk Analysis. Chichester: John Wiley \& Sons, 2003. 190 p. http://dx.doi. org/10.1002/0470871245

AZADEH, A. et al. Design and implementation of a fuzzy expert system for performance assessment of an integrated health, safety, environment (HSE) and ergonomics system: The case of a gas refinery. Information Sciences, v. 178 , p. $4280-4300.2008$. http://dx.doi.org/10.1016/j. ins.2008.06.026

BELLMAN, R. E.; ZADEH, L. A. Decision-Making in a Fuzzy Environment. Management Science, v. 17, p. B141-B164, 1970.

CAMBRAIS, F. B.; SAURIN, T. A.; FORMOSO, C. T. Planejamento e controle integrado entre segurança e produção em processos críticos na construção civil. Produção, v. 18, n. 3, p. 479-492, 2008.

CARR, V.; TAH, J. H. M. A fuzzy approach to construction project risk assessment and analysis: construction project management risk systems. Advances in Engineering Software, v. 32, p. 847-857, 2001. http://dx.doi. org/10.1016/S0965-9978(01)00036-9

CHOUDHRY, M.; FANG, D.; MOHAMED, S. The nature of safety culture: A survey of the state-of-the-art. Safety Science, v. 45, p. 993-1012, 2007. http://dx.doi. org/10.1016/j.ssci.2006.09.003

COOPER, D. F. et al. Project risk management guidelines: managing risk in large projects and complex procurements. Chichester: John Wiley \& Sons, 2006. p. 384.

CORNELL, M. E. P. Uncertainties in risk analysis: Six levels of treatment. Reliability Engineering and System Safety, v. 54, p. 95-111, 1996. http://dx.doi.org/10.1016/ S0951-8320(96)00067-1

CORREA, C. R. P.; CARDOSO JUNIOR, M. M. Análise e classificação dos fatores humanos nos acidentes industriais. Produção, v. 17, n. 1, p. 186-198, 2007. http://dx.doi.org/10.1590/S0103-65132007000100013

DAGDEVIREN, M.; IHSAN, Y. Developing a fuzzy analytic hierarchy process (AHP) model for behavior-based safety management. Information Sciences, v. 178, p. 1717-1733; 2008. http://dx.doi.org/10.1016/j. ins.2007.10.016

DEDOBBELEER, N.; BELAND, F. A Safety Climate Measure for Construction Sites. Journal of Safety Research, v. 22, p. 97-103, 1991. http://dx.doi. org/10.1016/0022-4375(91)90017-P

DONG, X. et al. Medical Costs and Sources of Payment for Work-Related Injuries Among Hispanic Construction Workers. Journal of Occupational and Environmental Medicine, v. 12, n. 49, p. 1367-1375, 2007. PMid:18231083. http://dx.doi.org/10.1097/ J0M.0b013e31815796a8

DUARTE, F. J. C. M.; CORDEIRO, C. V. C. A etapa de execução da obra: um momento de decisões. Produção, v. 9, numero especial, p. 5-27, 1999.

FABER, M. H.; STEWART, M. G. Risk assessment for civil engineering facilities: critical overview and discussion. Reliability Engineering and System Safety, v. 80, p. 173-184, 2003. http://dx.doi.org/10.1016/ S0951-8320(03)00027-9 
FREJ, T. A.; ALENCAR, L. A. Fatores de sucesso no gerenciamento de múltiplos projetos na construção civil em Recife. Produção, v. 20, n. 3, p. 322-334, 2010. http://dx.doi.org/10.1590/S0103-65132010005000043

GILLEN, M. et al. Injury severity associated with nonfatal construction falls. American Journal of Medicine, n. 32, p. 647-655, 1997.

GUERRINI, F. M.; VERGNA, J. R. G. Um modelo de atores e recursos para redes de cooperação entre empresas em obras de edificações. Produção, v. 21, n. 1, p. 14-26, 2011. http://dx.doi.org/10.1590/S0103-65132011005000009

GULDENMUND, F. W. The nature of safety culture: a review of theory and research. Safety Science, v. 34, n. 1-3, p. 215-257, 2000. http://dx.doi.org/10.1016/S09257535(00)00014-X

GURCANLI, G. E.; MUNGEN, U. An occupational safety risk analysis method at construction sites using fuzzy sets. International Journal of Industrial Ergonomics, v. 39, p. 371-387, 2009. http://dx.doi.org/10.1016/j. ergon.2008.10.006

HAMMER, W.; PRICE, D. Occupational Safety Management and Engineering. 5. ed. New Jersey: Prentice Hall Editors, 2001. 603 p.

HANSON, J. P.; REDFERN, M. S.; MAZUMDAR, M. Predicting slips and falls considering required and available friction. Ergonomics, v. 42, n. 12, p. 1619-1633, 1999. PMid:10643404. http://dx.doi. org/10.1080/001401399184712

HOLLNAGEL, E. Risk + barriers = safety? Safety Science, v. 46, p. 221-229, 2008. http://dx.doi.org/10.1016/j. ssci.2007.06.028

HYOUNG, J. et al. The characteristics of fatal occupational injuries in Korea's construction industry, 1997-2004. Safety Science, v. 47, n. 8, p. 1159-1162, 2009.

INSTITUTO NACIONAL DE ESTATÍSTICA - INE. Statistical Yearbook of Portugal 2007. Lisboa: INE 2008. 622 p.

KANGARI, R.; RIGGS, L. S. Construction Risk Assessment by Linguistics. IEEE Transactions on Engineering Management, v. 36, n. 2, 1989. http://dx.doi. org/10.1109/17.18829

KARWOWSKI, W.; MITAL, A. Potential Applications of Fuzzy Sets in Industrial Safety Engineering. Fuzzy Sets and Systems, v. 19, p. 105-120, 1986. http://dx.doi. org/10.1016/0165-0114(86)90031-X

KENTEL, E.; ARAL, M. M. Probabilistic-fuzzy health risk modelling. Stochastic Environmental Research and Risk Assessment, v. 18, p. 324-338, 2004. http://dx.doi. org/10.1007/s00477-004-0187-3

KOHN, M. C. Achieving credibility in risk assessment models. Toxicology Letters, v. 79, p. 107-114, 1995. http:// dx.doi.org/10.1016/0378-4274(95)03362-0

LAITINEN, H.; MARJAMAKI, M.; PAIVARINTA, K. The validity of the TR safety observation method on building construction. Accident Analysis and Prevention, v. 31, p. 463-472, 1999. http://dx.doi.org/10.1016/ S0001-4575(98)00084-0

LANDEWEERD, J. A. et al. Risk taking tendency among construction workers. Journal of Occupational Accidents, v. 11, p. 183-196, 1990. http://dx.doi. org/10.1016/0376-6349(90)90028-T

LEE, S.; HALPIN D. W. Predictive Tool for Estimating Accident Risk. Journal of Construction Engineering and
Management, v. 129, n. 4, p. 431-436, 2003. http:// dx.doi.org/10.1061/(ASCE)0733-9364(2003)129:4(431)

LIU, J. et al. Fuzzy Rule-Based Evidential Reasoning Approach for Safety Analysis. International Journal of General Systems, v. 33, n. 2-3, p. 183-204, 2004. http://dx.doi.or $\mathrm{g} / 10.1080 / 03081070310001633536$

LOOSEMORE, M.; LEE, P. Communication problems with ethnic minorities in construction industry. International Journal of Project Management, v. 20, p. 517-524, 2001. http://dx.doi.org/10.1016/S0263-7863(01)00055-2

LOOSEMORE, M. et al. Risk Management in Projects. 2. ed. London: Taylor \& Francis, 2006. 260 p.

McCAULEY-BELL, P.; BADIRU, A. Fuzzy Modeling and Analytic Hierarchy Processing to Quantify Risk Levels Associated with Occupational Injuries Part 1: The Development of Fuzzy Linguistic Risk Levels. IEEE Transactions on Fuzzy Systems, 1996.

MEARNS, K. J.; FLIN, R. Assessing the state of organizational safety - culture or climate? Current Psychology, v. 18, n. 1, p. 5-17, 1999. http://dx.doi.org/10.1007/ s12144-999-1013-3

MELIÁ, J. L. et al. Safety climate responses and the perceived risk of accidents in the construction industry. Safety Science, v. 46, n. 6, p. 949-958, 2008. http://dx.doi. org/10.1016/j.ssci.2007.11.004

MURE, S.; DEMICHELA, M.; PICCININI, N. Assessment of the risk of occupational accidents using a fuzzy approach. Cognition, Technology \& Work, v. 8, p. 103-112, 2006. http://dx.doi.org/10.1007/s10111-005-0025-5

NILSEN, T.; AVEN, T. Models and model uncertainty in the context of risk analysis. Reliability Engineering and System Safety, v. 79, 309-317, 2003. http://dx.doi. org/10.1016/S0951-8320(02)00239-9

NUNES, 1. L. Modelo de sistema pericial difuso para apoio à análise ergonómica de postos de trabalho. 2003. Tese (Doutorado em Engenharia Mecânica e Industrial)-Faculdade de Ciências e Tecnologia, Universidade Nova de Lisboa, Almada, Portugal, 2003.

NUNES, 1. L. Fuzzy Multicriteria Model for Ergonomic workplace analysis and Risk analysis. In: INTERNATIONAL CONFERENCE: COMPUTER-AIDED ERGONOMICS AND SAFETY - CAES'05, 2005, Kosice, Slovak Republic. Proceedings... Kosice, 2005.

OKE, S. A. et al. A fuzzy safety control framework for oil platforms. International Journal of Quality \& Reliability Management, v. 23, n. 5, p. 564-582, 2006. http:// dx.doi.org/10.1108/02656710610664613

PENDER, S. Managing incomplete knowledge: Why risk management is not sufficient. International Journal of Project Management, v. 19, p. 79-87, 2001. http:// dx.doi.org/10.1016/S0263-7863(99)00052-6

RINGDAHL, L. H. Safety Analysis principles and practice in occupational safety. 2. ed. London: Taylor \& Francis, 2001. 294 p.

RINGEN, K. et al. Why construction is different. Occupational Medicine, v. 19, n. 2, p. 255-259, 1995.

RU, W. G.; ELOFF, J. H. P. Risk analysis modelling with the use of fuzzy logic. Computers \& Security, v. 15, n. 3, p. 239-248, 1996. http://dx.doi. org/10.1016/0167-4048(96)00008-9

SAURIN, T. A. Segurança no trabalho e desenvolvimento de produto: diretrizes para integração na construção civil. 
Produção, v. 15, n. 1, p. 127-141, 2005. http://dx.doi. org/10.1590/S0103-65132005000100011

SAURIN, T. A.; RIBEIRO, J. L. D. Segurança no trabalho em um canteiro de obras: percepções dos operários e da gerência. Produção, v. 10, n. 1, p. 05-17, 2000.

SAWACHA, E.; NAOUM, S.; FONG, D. Factors affecting safety performance on construction sites. International Journal of Project Management, v. 17, n. 5, p. 309-315, 1999. http://dx.doi.org/10.1016/S0263-7863(98)00042-8

SII, H. S.; WANG, J.; RUXTON, T. Novel risk assessment techniques for maritime safety management system. International Journal of Quality \& Reliability Management, v. 18, n. 8-9, p. 982-999, 2001. http:// dx.doi.org/10.1108/02656710110407145

SILVERSTEIN, B. et al. Claims incidence of work-related disorders of the upper extremities: Washington state, 1993-1999. American Journal of Public Health, v. 88, p. 1827-1833, 1998. PMid:9842381. PMCid:1509055. http://dx.doi.org/10.2105/ AJPH.88.12.1827

TAM, C. M.; ZENG, S. X.; DENG, Z. M. Identifying elements of poor construction safety management in China. Safety Science, v. 42, p. 569-586, 2004. http://dx.doi. org/10.1016/j.ssci.2003.09.001

TIXIER, J. et al. Review of 62 risk analysis methodologies of industrial plants. Journal of Loss Prevention in the Process Industries, v. 15, p. 291-303, 2002. http://dx.doi. org/10.1016/S0950-4230(02)00008-6

TRBOJEVIC, V. M. Optimising hazard management by workforce engagement and supervision. London: Health and Safety Executive, 2008. 92 p. Research Report RR637.
WANG, H.; MCCAULEY-BELL, P. Fuzzy regression analysis to predict risk of occupational injuries. Fuzzy Sets and Systems, v. 92, n. 3, p. 317-340, 1997.

YANG, Z.; BONSALL, S.; WANG, J. Fuzzy Rule-Based Bayesian Reasoning Approach for Prioritization of Failures in FMEA. IEEE Transactions on Reliability, v. 57, n. 3, p. 517-528, 2008. http://dx.doi.org/10.1109/ TR.2008.928208

ZADEH, L. A. Fuzzy sets. Information and Control, v. 8, p. 338-353, 1965. http://dx.doi.org/10.1016/ S0019-9958(65)90241-X

ZADEH, L. A. Linguistic variables, approximate reasoning and dispositions. Informatics for Health and Social Care, v. 8, n. 3, p. 173-186, 1983. http://dx.doi. org/10.3109/14639238309016081

ZADEH, L. A. PRUF-a meaning representation language for natural languages. International Journal of Man-Machine Studies, v. 10, p. 395-460, 1978. http:// dx.doi.org/10.1016/S0020-7373(78)80003-0

ZADEH, L. A. Test-score semantics as a basis for a computational approach to the representation of meaning. Literary \& Linguistic Computing, v. 1 p. 24-35, 1986. http://dx.doi.org/10.1093/llc/1.1.24

ZIMMERMAN, H. J. Fuzzy Set Theory and its Applications. 2. ed. London: Kluwer Academic Publishers, 1993. 399 p.

\section{Agradecimentos}

Este estudo foi financiado pela Fundação para a Ciência e Tecnologia, bolsa n. ${ }^{\circ}$ SFRH / BD / 39610 / 2007.

\title{
Preliminary application of QRAM method for safety risk assessment in civil construction
}

\begin{abstract}
Risk Assessment for Health and Safety (RAH\&S) of workers is a complex task that involves the consideration of many parameters that are, quite often, difficult to quantify. RAHES in the construction industry is filled with inadequate data and/or imprecise and incomplete information, particularly in the design stage - to which traditional quantitative approaches do not have adequate answers. This study outlines the basic aspects for a Qualitative Risk Assessment Model (QRAM) based on elicited data, using fuzzy logic approach. The model was pre-validated in two Brazilian municipalities: Bauru, in the State of Sao Paulo, and Curitiba, in the State of Parana.
\end{abstract}

\section{Keywords}

Risk assessment. Occupational safety. Fuzzy sets. Construction industry. Validation. 
Anexo 1. Questionário.

Exm. ${ }^{\circ}$ Senhor

0 meu nome é Abel Pinto e estou desenvolvendo um projeto de pesquisa da minha tese de doutoramento na área da Ergonomia, a qual ocorre na Faculdade de Ciências e Tecnologia da Universidade Nova de Lisboa, sob a orientação da professora lsabel Nunes e da professora Rita Almeida. 0 estudo tem como objetivo o desenvolvimento e validação de uma metodologia para análise e avaliação de riscos para a segurança ocupacional (AARSO) quanto à segurança do trabalho, a qual pretende-se aplicar no setor da construção civil.

Atualmente estou em estágio de dois meses Programa de Pós-graduação em Design da Unesp / Campus Bauru, o qual é credenciado junto à CAPES/MEC e integra o Sistema Nacional de Pós-Graduação, sob supervisão do Prof. Dr. Luis Carlos Paschoarelli (http://lattes.cnpq. br/8521603444193259).

Esta investigação tem por objetivo a construção de um modelo de avaliação da qualidade do processo de avaliação dos riscos laborais. Assim, torna-se essencial ter o conhecimento amplo de como as empresas brasileiras do setor atualmente desenvolvem a área da análise e avaliação de riscos para a segurança ocupacional (AARSO) quanto à segurança do trabalho.

Considerando que vossa empresa é uma das referências no âmbito nacional, tomo a liberdade de me dirigir a vós no sentido de me auxiliar com essa abordagem, fornecendo informações às quais, de antemão já explicito, serão sempre tratados de forma anônima, global e estritamente com a finalidade acadêmica/científica.

Toda a informação da pesquisa será mantida confidencial e vou assumir toda a responsabilidade por quaisquer conclusões derivadas da análise de dados do inquérito.

Para qualquer esclarecimento suplementar contatar Abel Pinto: abel.fnpinto@clix.pt.

A sua resposta é importante. OBRIGADO.

Q1 - Caracterização do especialista

Função atual:

2. Experiência na função:

anos

3. Experiência na construção civil:

4. Está (ou esteve) envolvido com:

Realizar a AARSO $\square \quad$ Implementar a AARSO $\square \quad$ Avaliar a AARSO $\square$

Q2 - Caracterização da empresa

1. Dimensão:

Micro ( $<=10$ trabalhadores) $\square \quad$ Pequena (11 a 50 trabalhadores) $\square$

Média (51 a 200 trabalhadores) $\square \quad$ Grande (> 200 trabalhadores) $\square$

2. Tipo de obras que constrói (nesta questão pode escolher várias respostas):

Edifícios (habitação, fabris e outros) $\square \quad$ Infraestruturas (incluindo estradas) $\square \quad$ Obras de arte $\square \quad$ Obras marítimas ou ferroviárias $\square$ Outras $\square$ Quais?

Q3 - Caracterização do processo AARSO

1. Quem efetua (normalmente) a AARSO:

Coordenador de segurança de projeto $\square$ Técnico de HST $\square$ Diretor de obra $\square$

Outro $\square$ Qual?

2. Que metodologia(s) utiliza (nesta questão pode escolher várias respostas):

Análise preliminar de riscos $\square$ Método das matrizes $\square$ Método das energias $\square$ HAZOP $\square$ Árvore de falhas $\square$

Análise de tarefas $\square$ Análise de seg. no trabalho $\square$ Auditorias $\square$ Análise de desvios $\square$ Outra $\square$ Qual?

3. Como tomou conhecimento da metodologia utilizada:

Módulo do curso de HST $\square \quad$ Formação específica $\square$

Autodidata $\square$ Outro $\square$ Qual?

4. Conhece bem a metodologia utilizada:

$\mathrm{S} \square \mathrm{N} \square$

5. Conhece outras metodologias além da utilizada habitualmente:

$\mathrm{S} \square \mathrm{N} \square$ Em caso afirmativo: Quais?

6.Que Informação utiliza para realizar a AARSO:

Informação dos materiais $\square$ Informação dos equipamentos $\square$ Dados do projeto $\square$ Literatura especializada $\square \quad$ Dados de sinistralidade da empresa $\square$

Dados de sinistralidade do setor $\square \quad$ Experiência do próprio $\square$

Experiência de colega(s) $\square$ Outra $\square$ Qual?

7. Quem lhe fornece a informação referente à obra (nesta questão pode escolher várias respostas):

Dono de obra $\square$ Projetista(s) $\square$ Diretor de obra $\square$ Outro $\square$ Qual?

8. No processo de elaboração da AR, tem normalmente a colaboração de outros colegas:

$\mathrm{S} \square \mathrm{N} \square$ Em caso afirmativo, quais as suas funções? 
Anexo 1. Continuação...

$$
\text { Q3 - Caracterização do processo AARSO }
$$

9. No processo de elaboração da AARSO, há consulta dos trabalhadores (de alguns):

$\mathrm{S} \square \mathrm{N} \square$ Em caso afirmativo, quais as suas funções?

10. Em que fase da obra efetua a primeira AARSO:

Antes de iniciar a montagem do canteiro $\square \quad$ No início da montagem do canteiro $\square$

No começo da obra $\square \quad$ A pedido da fiscalização $\square \quad$ Outra $\square$ Qual?

11. Efetua atualizações da AARSO inicial:

$\mathrm{S} \square \mathrm{N} \square$

12. Em caso afirmativo, com que periodicidade, ou em que situações:

Antes de iniciar uma nova fase da obra $\square$ Quando ocorre um acidente $\square$

A pedido da fiscalização/inspeção $\square$ A pedido do coordenador de segurança $\square$

A pedido do diretor de obra $\square$ Outra $\square$ Qual?

13. No processo de AARSO, são considerados dados psicossociais (consumo de álcool ou drogas, por exemplo): $\mathrm{S} \square \mathrm{N} \square$

em caso afirmativo, quais?

14. No processo de AARSO, são considerados dados ergonômicos e de aptidão para o trabalho (idade, estatura, formação, trabalhadores com incapacidades permanentes...):

$\mathrm{S} \square \mathrm{N} \square$

em caso afirmativo, quais?

15. Qual a forma de comunicar os riscos aos trabalhadores: Verbal $\square$ Escrita $\square$

Outra $\square$ Qual?

16. Qual a importância que os trabalhadores atribuem aos resultados da análise de riscos:

Muita $\square \quad$ Pouca $\square \quad$ Nenhuma $\square \quad$ Desconheço $\square$

17. Qual a importância que as hierarquias atribuem aos resultados da análise de riscos:

Muita $\square \quad$ Pouca $\square \quad$ Nenhuma $\square \quad$ Desconheço $\square$

\section{Q4 - Caracterização da eficácia do processo AARSO}

1.Como classificaria o método de AARSO que usa, quanto à sua praticabilidade (maior ou menor facilidade de aplicação):

Fácil de aplicar $\square$ Nem fácil nem difícil $\square \quad$ Difícil de aplicar $\square$

2. Como classificaria o método de AARSO que usa, quanto à sua eficácia (atingir o resultado desejado):

Eficaz $\square \quad$ Pouco eficaz $\square \quad$ Ineficaz $\square$

3. Como classificaria o método de AARSO que usa, quanto ao tempo despendido (na sua aplicação):

Rápido de aplicar (um par de horas) $\square \quad$ Pouco rápido de aplicar (um ou dois dias) $\square$

Lento de aplicar (mais de dois dias) $\square$

4. quando ocorrem acidentes, normalmente o(s) risco(s) que o(s) originaram haviam sido identificados no processo de análise de riscos: $\mathrm{S} \square \mathrm{N} \square$

5. em caso afirmativo, o que falhou no processo:

0 risco havia sido considerado aceitável e, por isso, não foi controlado $\square$

as medidas de controle não foram implementadas $\square$

as medidas de controle foram ineficazes $\square$ o risco não foi comunicado $\square$

Outras razões $\square$ quais?

6. em caso negativo, o que falhou no processo:

0 perigo não foi identificado por deficiência do método $\square$

0 perigo não foi identificado por falta de conhecimentos/experiência do técnico $\square$

0 perigo não foi identificado por falta de informação relevante $\square$

0 perigo não foi identificado por utilização de informação errônea $\square$

Outras razões $\square$ quais?

7. Qual ou quais as maiores dificuldades que a sua empresa sente no processo de AARSO:

Falta de dados/informação $\square$ Tempo despendido $\square$ Falta de colaboração das chefias $\square$

Insuficiência de recursos (falta de técnicos de SST) $\square$ complexidade da metodologia $\square$

Outras razões $\square$ quais?

8. Caso não sinta dificuldade, é decorrente da facilidade e eficiência da metodologia utilizada: $\mathrm{S} \square \mathrm{N} \square$ Outras razões $\square$ quais?

Q5 - Caracterização da eficácia do novo método QRAM

1. Como classificaria o novo método, QRAM, quanto à sua praticabilidade (maior ou menor facilidade de aplicação):

Fácil de aplicar $\square \quad$ Nem fácil nem difícil $\square \quad$ Difícil de aplicar $\square$

Observações: 
Anexo 1. Continuação...

$$
\text { Q5 - Caracterização da eficácia do novo método QRAM }
$$

2.1 Como classificaria o novo método, ORAM, quanto à sua eficácia (atingir o resultado desejado):

Eficaz $\square$ Pouco eficaz $\square \quad$ Ineficaz $\square$

Observações:

3. Como classificaria o novo método, QRAM, quanto ao tempo despendido (na sua aplicação):

Rápido de aplicar (um par de horas) $\square \quad$ Pouco rápido de aplicar (um ou dois dias) $\square$

Lento de aplicar (mais de dois dias) $\square$

Observações:

4. Baseado na sua experiência, considera que o novo método, QRAM, caracteriza com exatidão os riscos existentes no canteiro:

$\mathrm{S} \square \mathrm{N} \square$

Observações:

6. em caso negativo, o que falhou no processo:

Algum perigo não foi identificado por deficiência do método $\square$

Na sua opinião, por quê?

Algum perigo não foi identificado por falta de conhecimentos/experiência do técnico $\square$

Na sua opinião, por quê?

Algum perigo não foi identificado por falta de informação relevante $\square$

Na sua opinião, por quê?

Algum perigo não foi identificado por utilização de informação errônea $\square$

Na sua opinião, por quê?

Alguns riscos foram mal avaliados

Na sua opinião, por quê?

Alguns riscos foram mal caracterizados (dificultando a decisão acerca das barreiras de segurança necessárias) $\square$

Na sua opinião, por quê?:

Outras Deficiências $\square$ quais?

Q6 - Analise comparativa do novo método QRAM

1.Considera o novo método, QRAM (nesta questão pode escolher várias respostas):

Mais fácil de aplicar $\square$ Mais rápido de aplicar $\square$ Mais fiável $\square \quad$ (que os métodos que usa atualmente)

Outra vantagem $\square$ Qual?

2.0s resultados com o novo método, QRAM, são equivalentes aos obtidos pelas metodologias que habitualmente usa?

3.Considera os resultados obtidos com o novo método, QRAM, fiáveis (i. e., refletem os riscos reais do estaleiro)? 